\title{
Digital Skeletal Radiography
}

\author{
Holger Pettersson
}

$\mathbf{D}^{\mathrm{u}}$ URING RECENT YEARS there has been an increased interest for digitalization of conventional radiographic examinations, such as radiographic examination of the chest, skeleton, and gastrointestinal tract. The most used systems for such digital radiography are based on imaging plates or phosphorous storage screens. The following presentation will focus on our experience of digital skeletal radiography using phosphorous storage screens at the University of Lund during the last 7 years. ${ }^{1}$ In the period 1986 through 1994, we have used the following systems: Philips PCR-901 (Philips Medical Systems, Shelton, CT), Siemens Digiscan 7000 (Siemens Medical Systems, Iselin, NJ), Fuji/Siemens 7501 (Fuji Medical Systems, Tokyo, Japan), Fuji FCR AC1, Fuji FCR AC2, and Fuji FCR 9000.

\section{EXPERIENCE IN THE CLINICAL ROUTINE}

The general advantages with phosphorous screen radiography are well known: broad exposure ranges, free choice of data processing, and possibilities for cheaper film handling.

The advantages of the broad exposure range are clearly seen in the daily routine. There are almost no retakes anymore, and this is not only an advantage for the patient, it is also highly appreciated by the personnel, especially in an emergency situation. It has also proved possible to diminish the radiation dose considerably (up to $50 \%$ ) for most routine examinations.

The possibilities for image manipulation are mainly used in routine situations by the technicians to improve the image quality in examinations that are difficult from an exposure point of view (such as examinations through plaster, the lateral view of cervical/thoracic region, etc). As a routine, we do not report at the workstations. Therefore, most image manipulation at the workstations that is performed by the radiologists involves research projects.

The possibilities for cheaper film handling have not been tested because the whole department still is not digitized, and we still use hard copies for image storage.

The potential disadvantages of the system are also well known: limited spatial resolution, de- creased possibility for evaluation of bone density, and contrast artifacts.

The spatial resolution for the systems commercially available today ranges from 3.5 to 7 line pairs $/ \mathrm{mm}$, which means a pixel size of about 0.1 $\mathrm{mm}$. The workstations generally used operate in the range of $512 \times 512$ to $2,024 \times 2,024$ pixels. This spatial resolution has not been experienced as a practical problem in the daily routine.

The decreased possibility to evaluate bone density, as well as the possibility of contrast artifacts, also mean no practical problem in daily routine, if the observer is aware of the possibility of the accurrence of these phenomena.

\section{DIAGNOSTIC ACCURACY}

A large department of skeletal radiology will meet all types of pathology within the musculoskeletal system, including the following: fracture, degeneration, osteoarthrosis, infection, inflammation, tissue lesions, spinal lesions, prosthesis, metabolic disturbance, tumor, and dysplasia. For a detailed scientific analysis of the diagnostic accuracy using imaging plate systems, we have choosen a few of these entities, to be representative for the diagnostic accuracy within the musculoskeletal system as a whole: rheumatoid arthritis, metabolic disturbance, fracture.

To evaluate the diagnostic accuracy in inflammatory disease, a study of rheumatoid arthritis was performed. 2 A total of 70 hands from patients with RA were examined using both conventional film-screen combination and imaging plates. In each hand, 11 joints (pip, mcp joints and wrists) were evaluated by four independent observers. The evaluation was performed on (1) the film-screen examination, (2) imaging plate hard copy (both simulating con-

From the Department of Radiology, University of Lund, Lund, Sweden.

Address reprint requests to Holger Petterson, MD, Department of Radiology, University of Lund, S-22I 85, Lund, Sweden.

Copyright 101995 by W.B. Saunders Company

0897-1889/95/0801-1011\$3.00/0 
ventional film and contrast enhanced), and (3) at a workstation.

The existence of the following parameters were decided: soft-tissue swelling, periarticular osteopenia, erosions, and joint-space narrowing.

An ROC analysis of the results was performed. For soft-tissue swelling, erosions, and joint-space narrowing, no significant difference was found between conventional film, digital plate hard copy and workstation. For periarticular osteopenia, the conventional film was significantly better at the spatial resolution level 3.3 $\mathrm{lp} / \mathrm{mm}$, whereas no significant difference was found at $5.0 \mathrm{lp} / \mathrm{mm}$.

For evaluation of the accuracy in metabolic disturbance, hyperparathyroidism was chosen. One hundred patients' hands were examined, and the second and third fingers were evaluated. The evaluation situation was the same as described above, and the four independent observers evaluated the existence of subperiosteal resorption, intracortical resorption and endosteal resorption. The receiver-operating characteristic analysis showed no significant difference between conventional film, digital plates, hard copy, and workstations.

Similarly, 70 patients with scaphoid fractures were examined, and again, no significant difference was found.

These findings are generally in concordance with those few reports on diagnostic accuracy in digital skeletal radiography that have been published from other centers. ${ }^{3-5}$

Therefore, we feel confident that the diagnostic accuracy using imaging-plate systems in skeletal radiology are at least at the same level as if conventional film-screen combinations are used. Moreover, the imaging-plate systems open new and interesting pathways for research.

\section{REFERENCES}

1. Pettersson H, Aspelin P, Boijsen E, et al: Digital radiography of the spine, large bones and joints using stimulable phosphor. Early clinical experience. Acta Radiologica 29:267, 1988

2. Jónsson A, Borg A, Hannesson P, Herrlin K, Jonsson K, Sloth M, Pettersson H: Film-screen vs. digital radiography in rheumatoid arthritis of hands: a receiver operating characteristics (ROC) analysis. Acta Radiol (in press)
3. Bramble JM, Murphey MD: Comparison of digital and conventional musculoskeletal radiography: Observer performance study. Radiology 177:587, 1990 (letter)

4. Buckwalter KA, Braunstein EM: Digital skeletal radiography. AJR 158:1071, 1992

5. Murphey MD: Digital skeletal radiography: Spatial resolution requirements for detection of subperiosteal resorption. AJR 152:541, 1989 